\title{
A review of remdesivir for COVID-19 in pregnancy and lactation
}

\author{
Sarah C. J. Jorgensen ${ }^{1 *}$, Matthew R. Davis $\mathbb{D}^{2}{ }^{2}$ and Stephen E. Lapinsky ${ }^{3,4}$ \\ ${ }^{1}$ Department of Pharmacy, Mount Sinai Hospital, Toronto, ON, Canada; ${ }^{2}$ Department of Pharmacy, University of California Los Angeles \\ Ronald Reagan Medical Center, Los Angeles, CA, USA; ${ }^{3}$ Division of Respirology, Mount Sinai Hospital, Toronto, ON, Canada; \\ ${ }^{4}$ Interdepartmental Division of Critical Care Medicine, University of Toronto, Toronto, ON, Canada \\ ${ }^{*}$ Corresponding author. E-mail: sarah.jorgensen@sinaihealth.ca
}

\begin{abstract}
Mounting evidence suggests that pregnant people have an elevated risk of severe COVID-19-related complications compared with their non-pregnant counterparts, underscoring the need for effective prevention and treatment strategies. However, despite progress in innovative and flexible trial designs during the COVID-19 pandemic, regressive policies excluding pregnant and breastfeeding people from biomedical research persist. Remdesivir, a broad-spectrum antiviral, was the first drug licensed for the treatment of COVID-19, based on data showing it reduced the time to recovery in hospitalized patients. Pregnant and breastfeeding people were specifically excluded from all clinical trials of remdesivir in COVID-19, but data are accumulating from post-marketing registries, compassionate use programmes and case series/reports. In this review we synthesize these data and highlight key knowledge gaps to help inform clinical decision-making about its use in pregnancy and lactation.
\end{abstract}

\section{Background}

Over the last 18 months, much has been learned about the effects of COVID-19 on pregnancy and perinatal outcomes. The results are sobering; higher rates of severe disease and complications have been documented amongst pregnant people in both highincome countries and low- and middle-income countries. ${ }^{1-5}$ In addition, severe and critical COVID-19 in pregnancy has been associated with elevated rates of premature birth and neonatal mor-

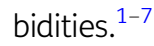

The management of pregnant people with COVID-19 presents unique clinical and ethical challenges, which are amplified by the relative scarcity of rigorous, high-quality data about safety and efficacy of interventions in this population. ${ }^{8,9}$ Despite a clear medical need, pregnant people continue to be routinely excluded from biomedical research, hampering efforts to provide evidence-based care. $^{9}$

Remdesivir is a nucleoside prodrug with broad-spectrum activity against human and zoonotic coronaviruses. ${ }^{10}$ It was the first drug licensed for the treatment of COVID-19 and has been shown to shorten the time to recovery in hospitalized patients requiring oxygen therapy. ${ }^{11}$ Its impact on mortality, however, is uncer$\operatorname{tain}^{11-15}$ and this uncertainty is reflected in the heterogeneity of consensus treatment guidelines, with some recommending for, and others against, its use in hospitalized patients with severe COVID-19. ${ }^{16-20}$

Pregnant and breastfeeding people were specifically excluded from all clinical trials evaluating remdesivir for COVID-19, ${ }^{11-15}$ and as such, there is no direct evidence of efficacy in this population. Pregnant people were eligible for an earlier Phase III trial evaluating remdesivir for Ebola virus infection; six pregnant patients were enrolled in the remdesivir arm $(3.4 \%$ of the participants who were randomized to remdesivir) and there were no significant maternal, fetal or newborn adverse events reported in this subgroup. ${ }^{21}$ Remdesivir has been used outside of clinical trials in pregnant people with COVID-19 and data on safety are accumulating from post-marketing registries, compassionate use programmes and case series/reports. ${ }^{22-33}$ The aim of this review was to summarize and synthesize these data to help inform clinical decision-making about remdesivir's use in pregnancy and lactation. To set the evidence in context, we briefly review background information on adverse pregnancy and fetal outcomes in general, and the effect that COVID-19 has on pregnancy and perinatal outcomes.

\section{Sources}

Literature searches of PubMed and Medline were conducted using various combinations of the search terms remdesivir, GS-5734, GS441524, pregnancy, lactation, breastfeeding, pharmacokinetics, COVID-19 and SARS-CoV-2. Reference lists of relevant articles were examined to identify sources not captured in the electronic literature search. Additional data were obtained from the US FDA regulatory approval review documents.

\section{Adverse pregnancy and fetal outcomes}

Adverse pregnancy outcomes include elective terminations, fetal losses, preterm birth ( $<37$ weeks) and maternal mortality. ${ }^{34,35}$ Fetal loss can result from spontaneous abortion, during the first 
20 weeks of gestation, or from stillbirth, after 20 weeks. ${ }^{34}$ Most spontaneous abortions occur in the first trimester as the result of chromosomal abnormalities. ${ }^{36}$ Global stillbirth rates show large disparities between developed and developing countries, with an estimated overall rate of 1.8\% (2015) but a range from $0.3 \%$ in developed regions to $2.9 \%$ in sub-Saharan Africa. ${ }^{37}$ Preterm births may be spontaneous or iatrogenic (i.e. medically indicated). The estimated global preterm birth rate in 2014 was $10.6 \%$, with significant differences by region, from $8.7 \%$ in Europe to $13.4 \%$ in North Africa. ${ }^{38}$ Maternal mortality refers to deaths due to complications of pregnancy or childbirth as well as deaths occurring within 42 days of pregnancy termination. ${ }^{35}$ It is standardized as the maternal mortality ratio, defined as the number of maternal deaths per 100000 live births during a given time period. Globally, the annual maternal mortality ratio was 211 in 2017 but varied widely from 5 in Western Europe to 533 in sub-Saharan Africa. ${ }^{35}$

Important adverse fetal outcomes include low birth weight $(<2.5 \mathrm{~kg})$ and birth defects. ${ }^{34}$ Low birth weight affects approximately $15 \%$ of live births globally and tracks with prematurity. ${ }^{39}$ The overall background prevalence of birth defects is between 3\% and $4 \%$, depending on the definition used, population studied and time period of follow-up. ${ }^{34,40}$ Birth defects secondary to drug exposure account for less than $1 \%$ of all birth defects. ${ }^{34}$ The embryonic period, between Weeks 3 and 8, marks the period of greatest sensitivity to the effects of teratogens. ${ }^{34}$

\section{Effect of COVID-19 on pregnancy outcomes}

Early in the COVID-19 pandemic it was unclear whether pregnancy was a risk factor for more severe illness. Initial data, mainly in the form of case series and centre experiences, were difficult to interpret, lacked appropriate comparator groups and were limited by other potential biases. Now that larger datasets are available, it is clear that pregnant people are at heightened risk of severe COVID19-related complications compared with their non-pregnant counterparts, particularly when infection occurs in the third trimester. ${ }^{1-5}$ For example, the risk of ICU admission and mechanical ventilation are approximately 2-4 times higher than those of nonpregnant populations of the same age. ${ }^{1,2,4}$ In addition, although the absolute risk of death in pregnant people with COVID-19 is low, it is many times higher than that of non-infected people of similar gestation. ${ }^{1,2}$ The majority of deaths have been concentrated in less-developed regions where comprehensive intensive care resources may be limited. ${ }^{2}$

The emergence of new variants has been linked to trends of increasing illness severity in pregnant and peripartum people, but a fulsome understanding of this association is still evolving. ${ }^{41}$ Similar to the general population, pre-existing comorbidities such as chronic hypertension, diabetes and obesity are additional risk factors for severe disease in pregnant people., ${ }^{1,2,5}$ The magnitude of risk to pregnant people is also amplified in minority populations. . $^{1,2,5}$

Multiple studies have shown that severe or critical COVID-19 infection during pregnancy increases the risk of preterm delivery and neonatal respiratory morbidity. ${ }^{1-7}$ The majority of preterm deliveries in severe and critical COVID-19 cases appear to be iatrogenic rather than due to direct effects of infection or maternal illness. ${ }^{42}$ In keeping with elevated rates of prematurity, neonatal ICU admissions and respiratory disorders have also been increased. ${ }^{1-5,7}$
Maternal SARS-CoV-2 infection has not been linked to birth defects, although data on infection in early pregnancy are limited. Some, but not all, studies have suggested that stillbirths are more common among pregnant people with COVID-19, although the numbers overall are low and estimates are imprecise. ${ }^{1-5,43}$

Data regarding intrauterine SARS-CoV-2 transmission have been reassuring. Although it has been reported ${ }^{44}$ intrauterine transmission appears to be rare. ${ }^{2,4,45}$ Similarly, transmission via breastmilk seems to be unlikely. ${ }^{46}$ Finally, when appropriate precautions are taken, the risk of mother-to-infant postnatal transmission appears to be low. ${ }^{47}$

\section{Preclinical data}

Remdesivir has been evaluated in in vitro and in vivo assays and was not found to exert genotoxic effects. For animal studies, remdesivir has been assessed in rat fertility and prenatal and postnatal studies, as well as rat and rabbit embryo-fetal developmental studies. ${ }^{48}$ In a prenatal study in rats, female rats exposed to remdesivir $10 \mathrm{mg} / \mathrm{kg} /$ day (1-fold exposure of regular human doses) exhibited a lower mean number of corpora lutea, and consequently lower mean numbers of implantation sites and viable embryos accompanied by lower mean ovary and uterus/cervix/ oviduct weights. These findings were considered to be related to remdesivir exposure and the 'no observed adverse effect level' (NOAEL) for female reproductive and embryonic toxicity was deemed to be $3 \mathrm{mg} / \mathrm{kg} / \mathrm{day}$, which provides approximately 0.34 fold exposure to regular human doses. No adverse effects on intrauterine growth, survival and fetal morphology were observed in embryo-fetal development studies in pregnant rats and rabbits with exposures up to four times higher than those achieved in humans with recommended dosing. ${ }^{48}$

Remdesivir transplacental transfer studies have not been completed; however, reasonable predictions can be made based on its chemistry and pharmacokinetics. Remdesivir is a monophosphoramidate prodrug; following IV administration it is rapidly hydrolysed by intracellular esterases to a nucleoside monophosphate analogue and requires several additional steps of metabolism to generate the active intracellular nucleotide triphosphate analogue. ${ }^{10}$ This suggests that remdesivir itself is unlikely to cross the placenta in clinically important amounts, but characteristics of major circulating metabolites, such as long half-lives, low molecular weights and high unbound fractions, ${ }^{10}$ suggest that they may have greater placental transfer.

\section{Clinical data}

Most of the published clinical experience with remdesivir in pregnant women comes from (1) Gilead's global safety database, which includes both Ebola virus and COVID-19 patients; and (2) the COVID-19 compassionate use programme (Table 1). ${ }^{23,32}$ It is possible that these sources included overlapping cases.

The global safety database included 156 cases of remdesivir exposure during pregnancy (cumulative to August 2020). ${ }^{32}$ Pregnancy outcomes were documented for less than one-third and included 33 live births and 13 adverse pregnancy outcomes ( 7 spontaneous abortions, 2 induced abortions and 4 stillbirths). Of the 13 cases with adverse pregnancy outcomes, 9 were reported in pregnant patients treated with remdesivir for Ebola virus 


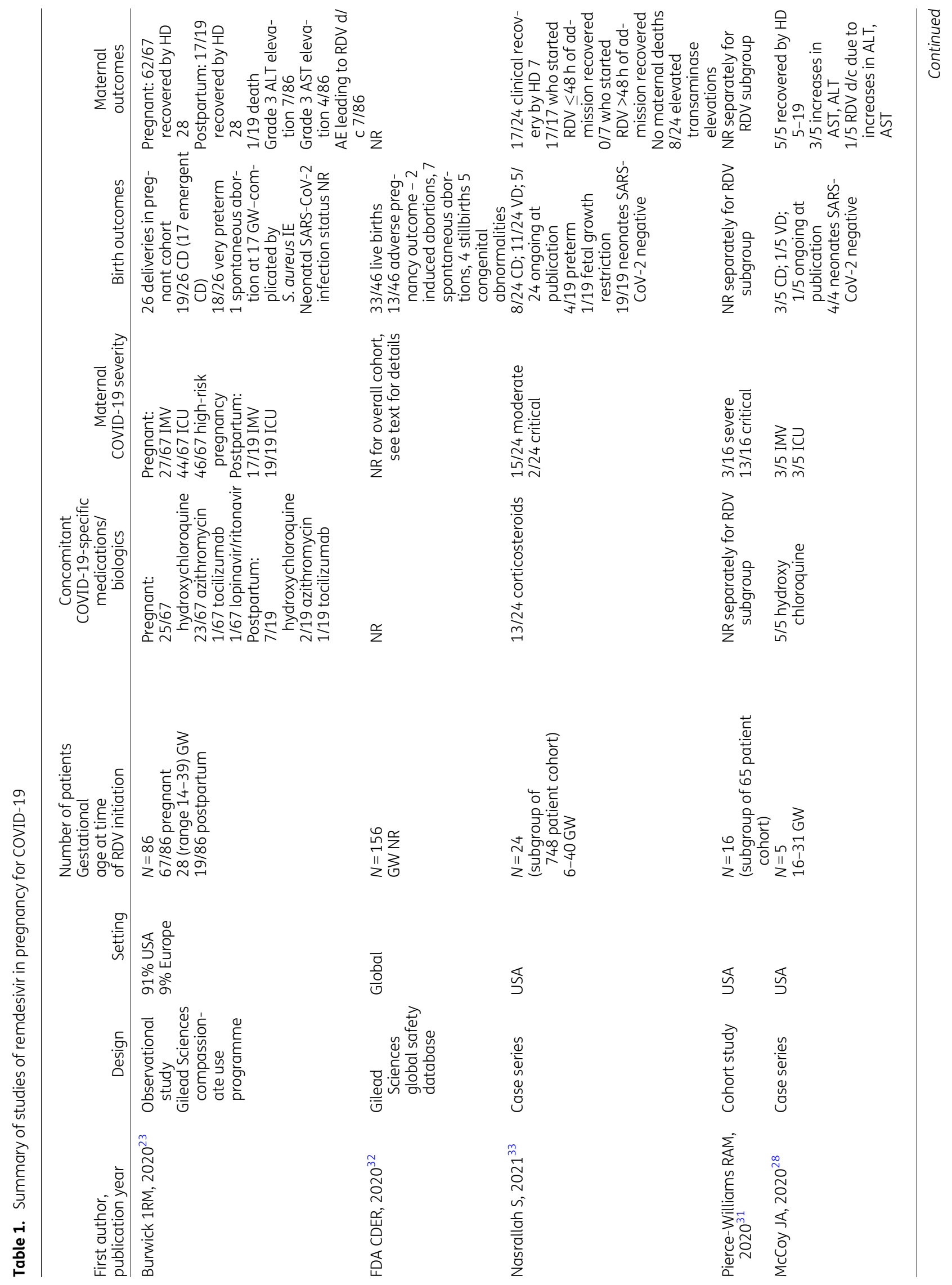




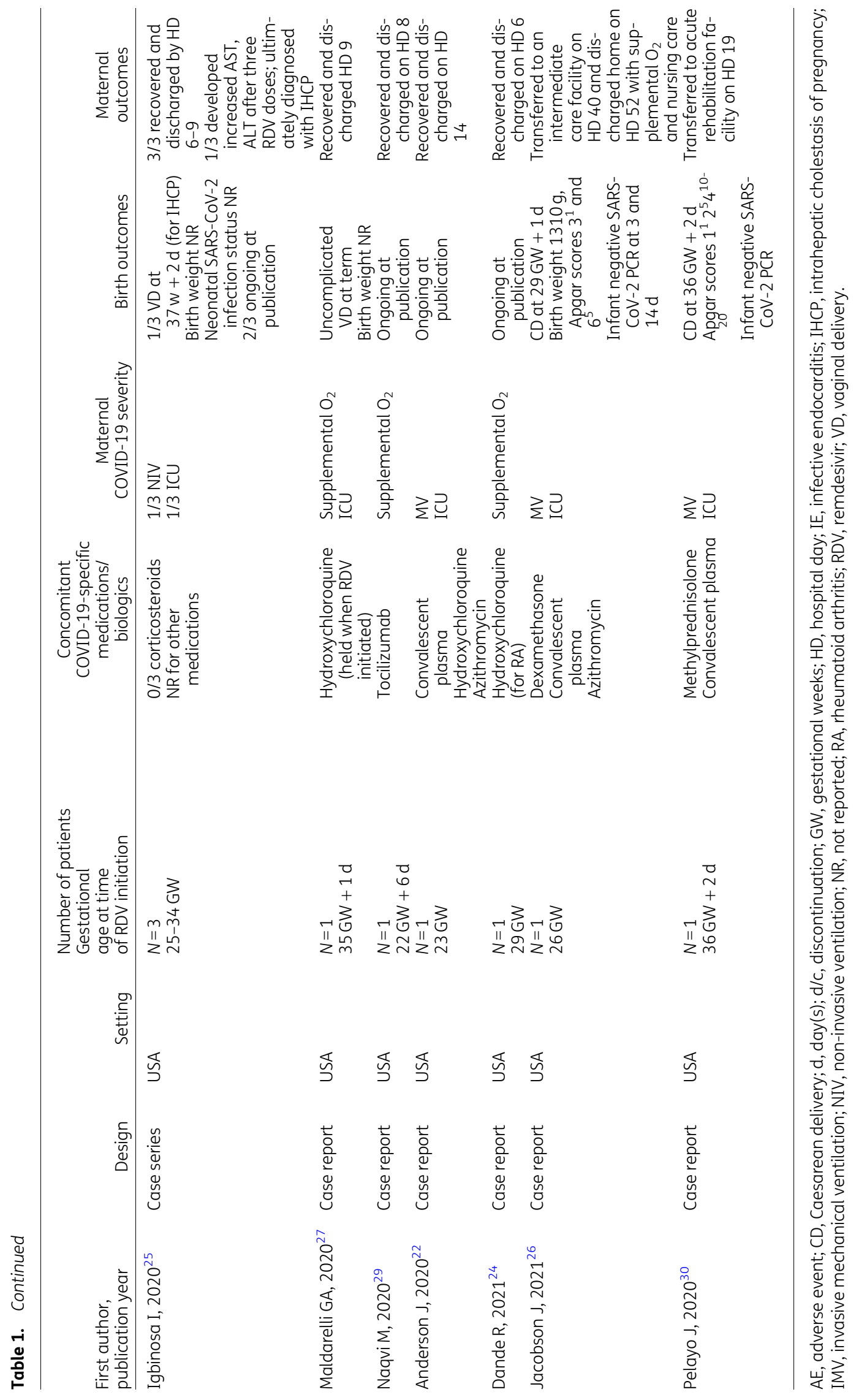


infection and 4 received remdesivir for COVID-19. All patients with COVID-19 who experienced adverse pregnancy outcomes were critically ill and required invasive mechanical ventilation within $24 \mathrm{~h}$ of remdesivir initiation. Five cases of congenital abnormalities were also identified; in all cases, remdesivir exposure occurred after the first trimester.

Sixty-seven pregnant people received remdesivir through the COVID-19 compassionate use programme during a 1 month period early in the pandemic (March-April 2020). ${ }^{23}$ Most (>90\%) were enrolled in the USA. The median gestational age was 28 weeks (range 14-39 weeks) with no first trimester exposures. A high proportion of critically ill pregnant patients were included: $67 \%$ were admitted to the ICU and $40 \%$ received invasive mechanical ventilation. Overall, 93\% recovered within 28 days. In keeping with results in non-pregnant patients, pregnant patients not requiring invasive mechanical ventilation had the highest rates of recovery $(98 \%)$ and the shortest median time to recovery (5 days). Amongst 26 deliveries, 69\% were delivered very preterm (24-32 weeks gestation). No neonatal deaths occurred during the observation period and there were no congenital abnormalities observed at birth (M. Das, Gilead Sciences; personal communication, 3 May 2021). There was one spontaneous abortion at 17 weeks' gestation in a woman with concurrent Staphylococcus aureus bacteraemia, endocarditis and septic arthritis and one maternal death after delivery, which was attributed to COVID-19.

The recovery rate in this report is reassuring, particularly considering the high prevalence of critical illness at baseline. ${ }^{23}$ Case series and single case reports also support rapid recovery in pregnant people treated with remdesivir for COVID-19, with most being discharged from hospital within 6-9 days (Table 1). ${ }^{22-25,27-29,33}$ However, without a control group the contribution of remdesivir to recovery is unclear.

The high rate of very preterm delivery reported in the compassionate use programme ${ }^{23}$ is troubling, but without an appropriate comparator group and contextual details it is difficult to determine whether there is a causal link with remdesivir. As noted previously, COVID-19 infection itself in pregnancy carries an elevated risk of preterm delivery, especially in the setting of critical illness. ${ }^{1-5}$ In addition, the rate of very preterm delivery in patients followed through this programme who received remdesivir postpartum was numerically similar (63\%) to those who received it during pregnancy. ${ }^{23}$ If nothing else, it is disappointing that this occurred despite the use of remdesivir, underscoring the need for more effective therapies in pregnant patients.

It is clear that published reports of remdesivir treatment in pregnant people lack many salient details (Table 1). ${ }^{22-33}$ For example, in the publications from the safety database and compassionate use programme, birth weight, the need for neonatal ICU admission and SARS-CoV-2 perinatal infections were not reported. ${ }^{23,32}$ Many of these outcomes were also missing in the other case series and reports outlined in Table 1 . The small number of documented negative PCR results in infants of mothers treated with remdesivir (Table 1) are insufficient to determine whether remdesivir can prevent rare cases of perinatal transmission.

Increases in transaminases have been described in both pregnant and non-pregnant individuals treated with remdesivir. ${ }^{10,23,25,28,33}$ The mechanism and clinical importance remain unclear. Nearly $40 \%$ of people enrolled in the compassionate use programme experienced treatment-emergent graded liver enzyme abnormalities but grade 3 abnormalities were uncommon (ALT, 5\%; AST, 9\%). ${ }^{23}$ These liver enzyme abnormalities might be related to remdesivir, COVID-19, other pregnancy-related causes (e.g. preeclampsia) or unrelated causes. In one report, a pregnant patient treated with remdesivir was ultimately diagnosed with intrahepatic cholestasis of pregnancy, ${ }^{25}$ highlighting the need to keep the differential open when pregnant patients show signs of liver injury.

\section{Pharmacokinetics}

Pregnancy is associated with multiple physiological, anatomical and biochemical changes that can dramatically impact the pharmacokinetics of antivirals, potentially leading to subtherapeutic exposure, unpredictable adverse effects or accelerated resistance. ${ }^{49}$ Remdesivir pharmacokinetic data were derived from healthy nonpregnant adult volunteers. Sparse pharmacokinetic results from three critically ill patients with COVID-19 have also been reported, ${ }^{50,51}$ but no data describing remdesivir pharmacokinetics in pregnancy are available. Simulation studies suggest pregnancyrelated increases in glomerular filtration rate and renal tubular secretion may increase elimination of remdesivir's major active extracellular metabolite. ${ }^{52}$ Additionally, pregnancy-related changes in plasma protein concentrations due to displacement from protein binding sites and volume expansion may alter unbound concentrations of remdesivir and its metabolites, ${ }^{52}$ potentially leading to more rapid clearance. Higher doses or more frequent administration may be required during pregnancy to match exposure seen in nonpregnant populations. However, the clinical importance of altered exposure is unclear since pharmacokinetic/pharmacodynamic efficacy and toxicity targets have not been established.

\section{Breastfeeding}

There are no data on the use of remdesivir in nursing mothers. Since remdesivir undergoes extensive first-pass metabolism, infants are unlikely to absorb clinically important amounts of remdesivir from breastmilk. ${ }^{53}$ However, systemic exposure to metabolites could result from breastfeeding. Reassuringly, no adverse effects were documented in the small number of infants who received remdesivir for treatment of Ebola virus infection or COVID-19. ${ }^{54,55}$

\section{Conclusions}

Pregnant people have been recognized as a medically complex population during infectious disease pandemics since the preantibiotic era and continue to be disproportionately impacted during the current pandemic. ${ }^{1-5}$ Prevention and treatment strategies are therefore critically important to improve maternal and neonatal outcomes. However, there has been little progress in the equitable inclusion of pregnant and breastfeeding people in biomedical research, forcing clinicians and their pregnant patients to weigh potential benefits and harms of interventions based on very little information. $^{8,9}$

To assist in this decision-making, we reviewed data on remdesivir in pregnancy and lactation from a variety of sources. Although our review does not reveal serious safety signals, it is clear that the available data have serious limitations and are insufficient to 
delineate the complete spectrum of potential adverse outcomes associated with exposure to remdesivir during pregnancy and lactation. Importantly, there are very few data on remdesivir exposure in the first trimester, when organogenesis occurs. In addition, requisite pharmacokinetic data in pregnancy are currently lacking. The role of remdesivir in preventing vertical or postnatal SARS-CoV2 transmission is also unclear. Diligent follow-up and documentation of outcomes in pregnant people with COVID-19 treated with remdesivir will be important moving forward. Greater regulatory push is clearly needed to ensure studies to obtain pregnancy data are expedited.

\section{Funding}

This review was carried out as part of our routine work.

\section{Transparency declarations}

S.C.J.J. has received speaker's honorarium from Sunovion, outside this work. M.R.D. has served as a consultant for Ligand Pharmaceuticals, Inc., outside this work. S.E.L. has received payments from Cambridge University Press and Sage Publications Inc., outside this work.

\section{References}

1 Allotey J, Stallings E, Bonet M et al. Clinical manifestations, risk factors, and maternal and perinatal outcomes of coronavirus disease 2019 in pregnancy: living systematic review and meta-analysis. BMJ 2020; 370: m3320.

2 Villar J, Ariff S, Gunier RB et al. Maternal and neonatal morbidity and mortality among pregnant women with and without COVID-19 infection: the INTERCOVID multinational cohort study. JAMA Pediatr 2021; e211050.doi: 10.1001/jamapediatrics.2021.1050.

3 Knight M, Ramakrishnan R, Bunch K et al. Females in hospital with SARSCoV-2 infection, the association with pregnancy and pregnancy outcomes: a UKOSS/ISARIC/CO-CIN investigation, 25 March 2021. https://www.gov.uk/gov ernment/publications/ukossisaricco-cin-females-in-hospital-with-sars-cov-2infection-the-association-with-pregnancy-and-pregnancy-outcomes-25march-2021.

4 Money D, Canadian Surveillance of COVID-19 in pregnancy: epidemiology, maternal and infant outcomes. Report \#2. 2021. http://med-fom-ridpro gram.sites.olt.ubc.ca/files/2021/01/CANCOVID_Preg-report-2-ON-AB-BC-QCdata_15JAN2021_FINAL.pdf.

5 Gurol-Urganci I, Jardine JE, Carroll F et al. Maternal and perinatal outcomes of pregnant women with SARS-CoV-2 infection at the time of birth in England: national cohort study. Am J Obstet Gynecol 2021; S0002-9378(21)00565-2. doi: 10.1016/j.ajog.2021.05.016.

6 Norman M, Naver L, Soderling J et al. Association of maternal SARS-CoV-2 infection in pregnancy with neonatal outcomes. JAMA 2021; 325: 2076-86.

7 Vouga M, Favre G, Martinez-Perez O et al. Maternal outcomes and risk factors for COVID-19 severity among pregnant women. Sci Rep 2021; 11: 13898.

8 Malhame I, D'Souza R, Cheng MP. The moral imperative to include pregnant women in clinical trials of interventions for COVID-19. Ann Intern Med 2020; 173: 836-7.

9 Taylor MM, Kobeissi L, Kim C et al. Inclusion of pregnant women in COVID19 treatment trials: a review and global call to action. Lancet Glob Health 2021; 9: e366-71.

10 Jorgensen SCJ, Kebriaei R, Dresser LD. Remdesivir: review of pharmacology, pre-clinical data, and emerging clinical experience for COVID-19. Pharmacotherapy 2020; 40: 659-71.
11 Beigel JH, Tomashek KM, Dodd LE et al. Remdesivir for the treatment of Covid-19 - final report. N Engl J Med 2020; 383: 1813-26.

12 Goldman JD, Lye DCB, Hui DS et al. Remdesivir for 5 or 10 days in patients with severe Covid-19. N Engl J Med 2020; 383: 1827-37.

13 Spinner CD, Gottlieb RL, Criner GJ et al. Effect of remdesivir vs standard care on clinical status at 11 days in patients with moderate COVID-19: a randomized clinical trial. JAMA 2020; 324: 1048-57.

14 Wang Y, Zhang D, Du G et al. Remdesivir in adults with severe COVID-19: a randomised, double-blind, placebo-controlled, multicentre trial. Lancet 2020; 395: 1569-78.

15 WHO Solidarity Trial Consortium, Pan H, Peto R et al.. Repurposed antiviral drugs for Covid-19 - interim WHO Solidarity trial results. N Engl J Med 2021; 384: 497-511.

16 NIH, COVID-19 Treatment Guidelines Panel. Coronavirus Disease 2019 (COVID-19) Treatment Guidelines. https://www.covid19treatmentguidelines. nih.gov/.

17 WHO. COVID-19 clinical management: living guidance. 25 January 2021. https://www.who.int/publications/i/item/WHO-2019-nCoV-clinical-2021-1.

18 NICE. COVID-19 rapid guideline: managing COVID-19 [NG191]. 2021. https://www.nice.org.uk/guidance/ng191/chapter/Recommendations.

19 National COVID-19 Clinical Evidence Taskforce. Clinical care living guidelines v39.1. https://covid19evidence.net.au/\#living-guidelines.

20 Bhimraj A, Morgan RL, Shumaker AH et al. Infectious Diseases Society of America guidelines on the treatment and management of patients with COVID-19. IDSA 2021; Version 4.3.0. https://www.idsociety.org/practiceguideline/covid-19-guideline-treatment-and-management/.

21 Mulangu S, Dodd LE, Davey RT Jr et al. A randomized, controlled trial of Ebola Virus Disease therapeutics. N Engl J Med 2019; 381: 2293-303.

22 Anderson J, Schauer J, Bryant $S$ et al. The use of convalescent plasma therapy and remdesivir in the successful management of a critically ill obstetric patient with novel coronavirus 2019 infection: a case report. Case Rep Womens Health 2020; 27: e00221.

23 Burwick RM, Yawetz S, Stephenson KE et al. Compassionate use of remdesivir in pregnant women with severe Covid-19. Clin Infect Dis 2020; ciaa1466. doi:10.1093/cid/ciaa1466.

24 Dande R, Qureshi A, Persaud K et al. Remdesivir in a pregnant patient with COVID-19 pneumonia. J Community Hosp Intern Med Perspect 2021; 11: 103-6.

25 Igbinosa I, Miller S, Bianco K et al. Use of remdesivir for pregnant patients with severe novel coronavirus disease 2019. Am J Obstet Gynecol 2020; 223: 768-70.

26 Jacobson J, Antony K, Beninati M et al. Use of dexamethasone, remdesivir, convalescent plasma and prone positioning in the treatment of severe COVID-19 infection in pregnancy: a case report. Case Rep Womens Health 2021; 29: e00273.

27 Maldarelli GA, Savage M, Mazur S et al. Remdesivir treatment for severe COVID-19 in third-trimester pregnancy: case report and management discussion. Open Forum Infect Dis 2020; 7: ofaa345.

28 McCoy JA, Short WR, Srinivas SK et al. Compassionate use of remdesivir for treatment of severe coronavirus disease 2019 in pregnant women at a United States academic center. Am J Obstet Gynecol MFM 2020; 2: 100164.

29 Naqvi M, Zakowski P, Glucksman L et al. Tocilizumab and remdesivir in a pregnant patient with coronavirus disease 2019 (COVID-19). Obstet Gynecol 2020; 136: 1025-9.

30 Pelayo J, Pugliese G, Salacup G et al. Severe COVID-19 in third trimester pregnancy: multidisciplinary approach. Case Rep Crit Care 2020; 2020: 8889487.

31 Pierce-Williams RAM, Burd J, Felder L et al. Clinical course of severe and critical coronavirus disease 2019 in hospitalized pregnancies: a United States cohort study. Am J Obstet Gynecol MFM 2020; 2: 100134. 
32 Center for Drug Evaluation and Research. Clinical Review. NDA 214787. Remdesivir (Veklury). 2020. https://www.accessdata.fda.gov/drugsatfda_ docs/nda/2020/2147870rig1s000MedR.pdf.

33 Nasrallah S, Nguyen AQ, Hitchings L et al. Pharmacological treatment in pregnant women with moderate symptoms of coronavirus disease 2019 (COVID-19) pneumonia. J Matern Fetal Neonatal Med 2021; 1-8.

34 Pregnancy Outcomes Working Group of the FDA Pregnancy Labeling Taskforce in the Center for Drug Evaluation and Research (CDER) and the Center for Biologics Evaluation and Research (CBER). Reviewer guidance: evaluating the risks of drug exposure in human pregnancies. 2005. https://www. fda.gov/media/71368/download.

35 UNICEF Data. Maternal Mortality 2000-2017. 2019. https://data.unicef. org/topic/maternal-health/maternal-mortality/.

36 Cunningham FG, Levono KJ, Bloom SL et al. Abortion. In: Seils A, Edmonson KG, Davis K, eds. Williams Obstetrics. McGraw-Hill, 2005; 231-251.

37 Blencowe H, Cousens S, Jassir FB et al. National, regional, and worldwide estimates of stillbirth rates in 2015, with trends from 2000: a systematic analysis. Lancet Glob Health 2016; 4: e98-108.

38 Chawanpaiboon S, Vogel JP, Moller AB et al. Global, regional, and national estimates of levels of preterm birth in 2014: a systematic review and modelling analysis. Lancet Glob Health 2019; 7: e37-46.

39 Blencowe $\mathrm{H}$, Krasevec J, de Onis $\mathrm{M}$ et al. National, regional, and worldwide estimates of low birthweight in 2015, with trends from 2000: a systematic analysis. Lancet Glob Health 2019; 7: e849-60.

40 CDC. Update on overall prevalence of major birth defects-Atlanta, Georgia, 1978-2005. MMWR Morb Mortal Wkly Rep 2008; 57: 1-5.

41 Kadiwar S, Smith JJ, Ledot S et al. Were pregnant women more affected by COVID-19 in the second wave of the pandemic? Lancet 2021; 397: 1539-40.

42 Metz TD, Clifton RG, Hughes BL et al. Disease severity and perinatal outcomes of pregnant patients with coronavirus disease 2019 (COVID-19). Obstet Gynecol 2021; 137: 571-80.

43 Khalil A, von Dadelszen P, Draycott T et al. Change in the incidence of stillbirth and preterm delivery during the COVID-19 pandemic. JAMA 2020; 324: 705-6.

44 Vivanti AJ, Vauloup-Fellous C, Prevot S et al. Transplacental transmission of SARS-CoV-2 infection. Nat Commun 2020; 11: 3572.
45 Woodworth KR, Olsen EO, Neelam V et al. Birth and infant outcomes following laboratory-confirmed SARS-CoV-2 infection in pregnancy - SET-NET, 16 jurisdictions, March 29-October 14, 2020. MMWR Morb Mortal Wkly Rep 2020; 69: 1635-40.

46 Chambers C, Krogstad P, Bertrand K et al. Evaluation for SARS-CoV-2 in breast milk from 18 infected women. JAMA 2020; 324: 1347-8.

47 Salvatore CM, Han JY, Acker KP et al. Neonatal management and outcomes during the COVID-19 pandemic: an observation cohort study. Lancet Child Adolesc Health 2020; 4: 721-7.

48 Center for Drug Evaluation and Research, Department of Health and Human Services, FDA. Veklury ${ }^{\mathrm{TM}}$ (Remdesivir [RDV]/GS-5734). Non-clinical pharmacology/toxicology NDA review and evaluation. 2020. https://www. accessdata.fda.gov/drugsatfda_docs/nda/2020/2147870rig1s000PharmR. pdf.

49 Costantine MM. Physiologic and pharmacokinetic changes in pregnancy. Front Pharmacol 2014; 5: 65.

50 Sorgel F, Malin JJ, Hagmann $\mathrm{H}$ et al. Pharmacokinetics of remdesivir in a COVID-19 patient with end-stage renal disease on intermittent haemodialysis. J Antimicrob Chemother 2021; 76: 825-7.

51 Tempestilli M, Caputi P, Avataneo V et al. Pharmacokinetics of remdesivir and GS-441524 in two critically ill patients who recovered from COVID-19. J Antimicrob Chemother 2020; 75: 2977-80.

52 International Maternal Pediatric Adolescent AIDS Clinical Trials Network (IMPAACT) 2032. Pharmacokinetics and Safety of Remdesivir for Treatment of COVID-19 in Pregnant and Non-Pregnant Women in the United States. Protocol version 2.0. 18 December 2020. https://www.impaactnetwork.org/sites/default/ files/2021-04/IMPAACT_2032_PROTOCOL_FINAL_V2.0_18DEC2020_CM_1.pdf.

53 Drugs and Lactation Database (LactMed). Bethesda (MD): National Library of Medicine (US). 2006. https://www.ncbi.nlm.nih.gov/books/ NBK501922/.

54 Frauenfelder C, Brierley J, Whittaker E et al. Infant with SARS-CoV-2 infection causing severe lung disease treated with remdesivir. Pediatrics 2020; 146: e20201701.

55 Dornemann J, Burzio C, Ronsse A et al. First newborn baby to receive experimental therapies survives Ebola Virus Disease. J Infect Dis 2017; 215: $171-4$. 